\title{
Adolf Reinach sur le continu
}

\author{
Denis Seron (FNRS, Univ. de Liège)
}

Philosophie, 128 (janv. 2016), p. 34-49.

Adolf Reinach a consacré de patientes et subtiles analyses au continu dans un cours du semestre d'hiver 1913-1914 et du semestre d'été 1914, intitulé Sur l'essence du mouvement ${ }^{1}$. Ce texte, qui compte certainement parmi ses plus difficiles d'accès, a pour point de départ et pour fil conducteur les apories de Zénon. Quoi que puisse faire penser son titre, les problèmes qui y sont abordés excèdent largement la seule question du mouvement. Cela pour la simple raison que les apories de Zénon elles-mêmes s'étendent loin au-delà de la question du mouvement ${ }^{2}$. Reinach observe ainsi, avec raison, qu'elles s'appliquent aussi au cas de variations intensives où la notion d'espace ne joue aucun rôle. Par exemple, la démonstration de l'impossibilité du mouvement par le nombre infini d'espaces intermédiaires est facilement transposable aux variations intensives : un son ne peut gagner en intensité, parce qu'il y a nécessairement un nombre infini de degrés intensifs intermédiaires entre son intensité initiale et celle qu'il est censé acquérir ( $W B$, p. 556). En réalité, l'enjeu des apories de Zénon est le changement continu. C'est celui-ci, et non le mouvement, qui forme le véritable thème du cours.

Reinach estime que les apories de Zénon nous donnent accès à une grande variété de problèmes qui sont au centre de la tradition philosophique dans son ensemble, depuis l'infini indénombrable jusqu'à l'opposition de l'être et du devenir, en passant par le temps et l'espace :

D’Aristote à Bergson, on ne compte plus les tentatives visant à invalider $<$ les arguments de Zénon> ou à présenter le sens le plus profond de leur validité. Les penseurs ont toujours de nouveau été séduits par l'idée de tester la valeur d'idées nouvelles et fondamentales par le fait qu'elles seules pourraient conduire ces paradoxes immémoriaux à une solution définitive. À cela s'ajoute que les recherches les plus pénétrantes consacrées aux problèmes de l'infini et du constant ont trouvé dans ces arguments leur point de départ et y ont eu recours. Une histoire des problèmes de Zénon ferait défiler devant nous une grande part de l'histoire de la philosophie à partir d'un point particulièrement stimulant. Elle donnerait un aperçu rare de la manière dont ces adorables petites histoires d'Achille et la tortue et de la flèche en repos forment, à travers les siècles, le centre des réflexions les plus significatives et les plus importantes. ( $W B$, p. 555.) 
Aussi le texte renferme-t-il d'importants développements sur des problèmes qui ne se rattachent qu'indirectement à celui du continu. Aux $\S \S 1$ et 2 , par exemple, Reinach soulève la question - centrale dans son réalisme et également posée, sous une forme à peine différente, dans l'Introduction à la philosophie de 1913 - de savoir si et dans quelle mesure on peut inférer un être ou un pouvoir-être de l'intuition.

Reinach avait antérieurement traité des apories zénoniennes dans des leçons sur Platon au cours du semestre d'été 1910, dont seules sont parvenues jusqu'à nous des notes d'Alexandre Koyré $^{3}$. D'après les éditeurs des Sämtliche Werke, il y avait opté pour une solution aristotélicienne, que le texte de 1913-1914 remettra en cause pour une grosse part. L'université de Göttingen, où Reinach enseignait comme Privatdozent depuis 1909 aux côtés de Husserl, était un lieu particulièrement propice à l'étude du continu comme des paradoxes logiques et mathématiques. Zermelo y avait étudié et enseigné jusqu'en 1910, comme avant lui Dedekind. Leonard Nelson et Kurt Grelling, auteurs de l'antinomie homonyme, y menaient des recherches à cette période. Le mathématicien et physicien Hermann Weyl y avait fait ses études, avant d'y enseigner comme Privatdozent de 1910 à 1913. Son ouvrage de 1918 sur le continu, influencé par Husserl, discute le paradoxe de Nelson et Grelling dans le cadre de sa fondation intuitive de l'analyse ${ }^{4}$. Bien que les échanges soient mal attestés, il est plausible que la proximité a dû stimuler les phénoménologues rassemblés autour de Husserl et Reinach à mener leurs propres recherches sur ce genre de questions. Husserl a amené son élève Hans Lipps à rédiger sa thèse sur les antinomies logiques. Alexandre Koyré s'est intéressé aux apories de Zénon dans sa thèse refusée par Husserl en 1911, d'où il a tiré une étude publiée en 1922 dans le Jahrbuch, d'ailleurs dédiée à Reinach ${ }^{5}$.

Le but des analyses qui suivent est de clarifier le texte sur le mouvement de Reinach, en en dégageant les principales lignes argumentatives. Ce qui permettra de jeter quelques lumières sur une conception originale et profonde de ce que signifie devenir - une conception que, curieusement, on ne trouve nulle part ailleurs exprimée sous cette forme dans l'œuvre conservée de Reinach. Je commencerai par esquisser quelques idées maîtresses du cours sur le mouvement, avant d'en tirer les conséquences s'agissant des apories de Zénon.

\section{L'essence du mouvement}

Reinach considère que la recherche mathématique n'est d'aucune aide pour résoudre les apories zénoniennes, du moins aussi longtemps qu'elle n'a pas été préparée par la recherche philosophique, c'est-à-dire par un travail d'idéation et de clarification des essences ( $W B$, 
p. 555). Bien que Reinach ne l'eût certainement pas avalisé, le terme de « grammaire » (du devenir) conviendrait assez bien à ce dernier type de recherches.

Le cours sur le mouvement s'ouvre sur une sorte d'introduction polémique où Reinach s'en prend principalement aux interprétations aristotélicienne et bergsonienne des apories de Zénon, ainsi qu'aux théories du mouvement de Berkeley et de Paul Ferdinand Linke. À l'exception de la critique de Bergson, sur laquelle il faudra revenir, ces développements sont périphériques et peuvent n'être évoqués que sommairement.

L'objection que Reinach adresse à Aristote est d'avoir restreint erronément la portée des apories de Zénon et, en conséquence, d'en avoir proposé une solution insuffisamment puissante. Comme on l'a suggéré, les apories de Zénon s'étendent à toutes sortes de devenirs qui ne sont pas des mouvements, par exemple aux gradations intensives. C'est pourquoi, déclare Reinach, « les problèmes relatifs à l'espace sont inessentiels » ( $W B$, p. 556). Il est vain d'objecter à Zénon que l'espace et le temps, bien que divisibles à l'infini, ne sont pas pour autant actuellement composés. Ce n'est pas simplement la structure de l'espace et du temps qui doit être clarifiée, mais celle du continu. Le vrai problème, nous dit Reinach, est celui du « changement continu en général » $(W B$, p. 556).

La critique de Bergson est plus complexe. Pour l'essentiel, celui-ci a commis une double erreur. D'abord, il a interprété les apories de Zénon comme si le mouvement de $A$ à $B$ s'interrompait en chaque point entre $A$ et $B$. Or, Reinach estime que cette interprétation n'est pas fidèle à l'idée de Zénon. Ensuite, Bergson a cru pouvoir résoudre les apories en ramenant le mouvement à un processus mental indivisible. Mais cette idée est absurde : le mouvement a lieu dans les choses, il est parfaitement étranger aux activités de l'ego (WB, p. 559-560) ${ }^{6}$.

Reinach défend au $\S 3$ une thèse centrale qui détermine profondément son analyse du mouvement : tout mouvement est par essence absolu. Il oppose cette thèse, à première vue surprenante, non seulement à Berkeley, mais aussi à la toute récente théorie de la relativité. Son argument, assez simple et présent sous différentes formes partout dans le texte sur le mouvement, consiste à distinguer entre d'une part ce que le mouvement est « en soi », du point de vue de ses déterminations « ontiques », et d'autre part ce qu'il est du point de vue de la « saisie » (Auffassung) qu'on peut en avoir. D'un côté, le mouvement a en soi certaines propriétés ; de l'autre, on peut « saisir » ou « établir » (feststellen) l'existence d'un mouvement. Ainsi, il est plausiblement impossible d'établir qu'un corps physique que je vois est absolument en mouvement ou un repos. Le quai que je vois par la fenêtre du train semble en mouvement alors qu'il est en réalité en repos ; ou bien il semble en repos tout en étant en mouvement relativement au centre de la terre, etc. De ce point de vue, il y a bien un sens à dire, comme Berkeley, que le mouvement « inclut nécessairement une relation ${ }^{7}$. Cependant, cela n'implique pas que tout mouvement est par essence relatif, ni n'exclut que tout mouvement soit par essence absolu : «Ce qui vaut de l'établissement (Feststellung) du 
mouvement et du repos dans le monde réel ne peut en aucune manière être transposé à l'essence du mouvement et du repos eux-mêmes. » (WB, p. 562.) Le mouvement comme le repos sont bien plutôt en soi des déterminations absolues des corps, qui cependant ne peuvent être établies ou saisies qu'en référence à d'autres corps.

À côté de son caractère absolu, Reinach développe au $\S 3$ une seconde caractéristique essentielle du mouvement, s'en prenant à la conception d'un autre élève de Husserl, Paul Ferdinand Linke ${ }^{8}$. En substance, Reinach conteste la thèse de Linke suivant laquelle le mouvement présuppose l'identité au sens où il n'est pas seulement par essence le mouvement de quelque chose - ce que Reinach reconnaît - mais aussi le mouvement de quelque chose d'identique. Il lui oppose l'idée que le mouvement implique certes la « mêmeté » (Selbigkeit) du mobile, mais non son Identität au sens du principe d'identité. D'où il consacre de riches analyses à la mêmeté et à son corrélat, l'« altérité » (Andersheit).

\section{2. Être et devenir}

On considère souvent que Reinach et à sa suite Ingarden sont à l'origine de la distinction, dans une large mesure inconnue de Husserl, entre acte et état mental. On réfère habituellement, sur ce point, à l'essai sur la négation de 1911, où Reinach reproche à Brentano de confondre l'état de conviction et l'acte d'affirmation ${ }^{9}$. On trouve un développement intéressant de cette distinction dans le texte sur le mouvement, où Reinach distingue clairement - comme le feront à sa suite Ingarden et Chisholm ${ }^{10}$ — entre d'une part les états et processus (Vorgänge) et, d'autre part, les points initiaux et finaux des états et processus.

Cette distinction se rattache à l'opposition entre être et devenir, à laquelle les leçons sur le mouvement consacre de minutieuses recherches. Prenons le mouvement d'un corps d'un point à un autre. Le mouvement lui-même est un processus, c'est-à-dire quelque chose qui « a lieu » (statthat), par opposition à l'état de repos qui lui préexistait et à la chose qui est en mouvement ( $W B$, p. 579). Par ailleurs, le mouvement a une certaine particularité que n'ont pas tous les processus : il est « constitutif d'un devenir ». Reinach exprime ce fait en disant qu'il n'est pas seulement un Vorgang, mais un Prozess (WB, p. 579). Ainsi, au moment où le mouvement commence, l'espace parcouru par le mouvement n'existe pas encore, puisqu'il n'a pas encore été parcouru. Cet espace n'est pas, mais il devient, c'est-à-dire qu'il naît et s'accroît continûment.

Pourtant, ajoute Reinach, le mouvement lui-même ne devient pas (WB, p. 580). Comme tout processus, le mouvement existe, et il commence à exister à un certain moment. De même, un 
état est quelque chose qui existe, comme l'est aussi une chose. La différence, dit Reinach de façon assez énigmatique, est qu'un processus est quelque chose de temporel, au sens de quelque chose qui « s'édifie dans le temps » (sich in der Zeit aufbaut). Par contre, les états et les choses sont des existants « intemporels » $(W B$, p. 580$)$.

Ces distinctions jouent un rôle déterminant dans l'analyse reinachienne des apories de Zénon, dont elles conditionnent toute solution d'ensemble. Les observations de Zénon apparaissent aporétiques précisément parce que la différence entre être et devenir est comprise incorrectement.

Revenons à la notion d'espace parcouru par un mobile, et supposons qu'un corps en mouvement de $A$ à $B$ passe par un point $C$. L'espace parcouru par le corps s'accroît continûment entre $A$ et $B$. En ce sens, il passe par plusieurs grandeurs différentes. Mais la question que se pose Reinach est de savoir si l'espace parcouru $a$ une grandeur déterminée lorsque le corps passe par le point $C$. À cette question, Reinach répond ceci : l'espace parcouru aurait une grandeur déterminée si le corps était au point $C$. Mais il n'y est pas, il passe par le point $C$ - ce qui est très différent. Car passer par un point, cela veut dire : le quitter ou y parvenir ( $W B$, p. 582). Or, au moment où le corps parvient au point $C$, l'espace parcouru n'a pas encore telle grandeur déterminée correspondant à $A C$, sinon il y serait déjà parvenu auparavant. De même, au moment où le corps quitte le point $C$, l'espace parcouru n'a déjà plus telle grandeur déterminée correspondant à $A C$, sinon il ne serait pas en train de quitter $C$. C'est pourquoi, dit Reinach, « ce qui grandit n' $a$ une grandeur à aucun instant du processus continu, mais il passe continûment par des grandeurs » (WB, p. 578).

Naturellement, cela ne veut pas dire que l'espace parcouru par le corps quand il passe par le point $C$ n'a aucune grandeur. La situation est plutôt celle-ci : l'espace parcouru a une grandeur, mais, ajoute Reinach, une grandeur qui n'est pas déterminée, ce qu'il dénomme un apeiron :

Ce qui est devenu jusqu'à tel ou tel moment du devenir, cela n'est pas saisissable comme une grandeur déterminée aussi longtemps que dure le devenir : c'est l'apeiron. Ce n'est que quand le devenir a cessé que le devenu devient déterminable. (WB, p. 580.)

En ce sens, Reinach estime que les apeira forment « une espèce particulière de grandeur à côté des grandeurs intensives et extensives » (WB, p. 583). (Ce qui n'est pas sans poser problème, dans la mesure où cela empêche de distinguer entre apeira intensifs et extensifs ${ }^{11}$.). La notion d'apeiron implique directement une autre distinction qui est cruciale dans le texte sur le mouvement, celle entre « emprunter » ou « couvrir » une distance et la « parcourir ». Supposons à nouveau qu'un corps se meuve d'un point $A$ à un point $B$ en passant par un point $C$. On a vu que la distance $A C$ n'était pas une grandeur déterminée, disons mesurable, mais un 
apeiron. Reinach dit en ce sens que la distance $A C$ est « parcourue » (durchlaufen, durchmessen). Mais on peut aussi considérer la distance $A B$ en supposant que le mouvement s'arrête au point $B$. Dans ce cas, le corps ne passe pas par $B$, mais l'espace $A B$ est une grandeur déterminée, mesurable, « fermée ». Dans la terminologie de Reinach, la distance $A B$ n'est pas « parcourue », mais « couverte » ou « empruntée » (zurückgelegt).

L'idée de Reinach est que, s'il prend fin au point $B$, alors le mouvement de $A$ à $B$ fait passer le corps par $A$ et par tous les points qui suivent, à l'exception du point $B$. Ce fait s'explique sans trop de difficultés. Le corps ne passe pas par $B$ simplement parce que si le mouvement prend fin en $B$, alors il est au point $B$ - c'est-à-dire qu'il y est en repos et non en mouvement. Au moment où le corps se trouve au point $B$, il n'est déjà plus en mouvement. Ainsi, si l'on veut être conséquent, on doit dire que le point $B$ n'est pas le dernier point de l'espace parcouru par le mouvement, mais le premier point où le corps est en repos. C'est pourquoi Reinach peut défendre la thèse en apparence paradoxale suivant laquelle l'espace parcouru, pendant tout le temps que dure le mouvement, n'est pas limité, c'est-à-dire limité par un point final ( $W B$, p. 580). La distinction entre « parcourir » et « couvrir » une distance est en fait superposable à celle entre apeiron et distance déterminée. Comme le résume Reinach, « les portions d'espace sont de l'espace (...) couvert, les apeira sont de l'espace parcouru » (WB, p. 583).

Le problème soulevé joue un rôle central dans l'approche reinachienne des apories de Zénon, comme il le faisait déjà dans la théorie brentanienne du continu. Lorsqu'un corps se met en mouvement en un point $A$ et s'arrête en un point $B$, est-il en repos ou en mouvement aux points $A$ et $B$ ? $A$ est le point où débute le mouvement, ce qui semble indiquer que le corps doit y être en mouvement ; mais il est aussi le point où prend fin le repos, d'où l'on est tenté de conclure que le corps doit y être en repos. Or un même corps ne peut être à la fois en mouvement et en repos. De même, $B$ est le point où prend fin le mouvement, ce qui suggère que le corps doit y être en mouvement; mais il est aussi le point où commence le repos, ce qui suggère que le corps doit y être en repos. Le problème est transposable, mutatis mutandis, au continuum temporel. Un corps en mouvement d'un temps $t$ à un temps $t$ ' est-il en repos ou en mouvement en $t$ et en $t$ '? Comme le mouvement commence en $t$, il semble que le corps doive y être en mouvement ; mais comme le repos s'achève en $t$, il semble aussi devoir y être en repos, etc.

Le problème, classique, était également posé par Brentano, mais les conclusions des deux auteurs sont dans une large mesure opposées. Brentano entendait résoudre le problème (et une multitude d'autres apparentés) au moyen de sa notion de limite d'un continuum ${ }^{12}$. Les instants $t$ et $t$ ', les points $A, B$ et $C$ ne sont pas - comme le voudrait l'analyse de Cantor et Dedekind, vivement critiquée dans les leçons sur le continu — des parties ponctuelles composant un continuum, mais des limites de continua. L'idée est que la continuité se définit par l'impossibilité de la contiguïté et par la possibilité de la coïncidence. D'une part, entre deux points donnés d'un continuum, il est toujours possible d'en introduire un troisième, ce 
qui signifie que deux points d'un continuum ne sauraient être contigus. D'autre part, un point est caractérisable comme une limite d'un ou plusieurs continua : $A$ et $B$ limitent le segment de droite $A B$; les deux segments $A C$ et $C B$ partagent une même limite $C$. Brentano spécifie cette propriété par la notion de "plérose » (Plerosis). $C$ possède une plérose complète tandis que $A$ et $B$, supposés limiter un unique segment, ont une demi-plérose relativement au segment. De même le segment $A B$ présente une demi-plérose relativement au triangle $A B C$, etc. Dans le cas qui nous occupe, cela signifie que le point ou l'instant auquel débute le mouvement peut sans contradiction être à la fois la limite de l'état de repos antérieur et de l'état de mouvement postérieur, et que le point ou l'instant auquel le mouvement prend fin peut sans contradiction être à la fois la limite de l'état de mouvement antérieur et de l'état de repos postérieur. Ainsi, « à l'instant auquel la chose commence à se mouvoir et aussi cesse d'être en repos, la chose est en mouvement. Et à l'instant auquel elle cesse de se mouvoir et commence à être en repos, elle est aussi en mouvement $»^{13}$.

La conception de Brentano présente pourtant des difficultés. Par exemple, la frontière entre l'Allemagne et l'Autriche doit être, dans la conception de Brentano, une limite commune aux deux territoires. Mais il peut sembler plus naturel de dire que ceux-ci n'ont simplement rien en commun ${ }^{14}$. La stratégie de Reinach, assez différente, permet d'éviter au moins partiellement ce genre de difficultés en s'appuyant non plus sur la notion de limite, mais sur la distinction entre être et devenir, entre être en un point et passer par un point. Sa thèse est que le mouvement est présent dès le point ou l'instant où il commence, mais qu'il ne peut y avoir de mouvement au point ou à l'instant où le mouvement s'arrête. Un corps en mouvement de $A$ à $B$ sera dit en mouvement au point $A$ où débute le mouvement et en tout point de $A B$ hormis $B$. En termes brentaniens, il est en mouvement s'il occupe un point de $A B$ dont la plérose est soit complète, soit incomplète vers l'antérieur.

Cette thèse se justifie aisément si l'on accepte les analyses qui précèdent. Qu'un corps soit en mouvement en tel ou tel point, suppose-t-on, cela implique qu'il passe par ce point. Or, il est impossible qu'un corps en mouvement de $A$ à $B$ passe par le point $B$, puisqu'il s'y arrête. Le corps passe par tous les points de $A B$ à l'exception de $B$. Ce qui implique que, rigoureusement parlant, la distance parcourue n'a pas de dernier point. Inversement, le corps sera dit en mouvement en $A$, et non en repos, puisqu'il ne fait qu'y passer sans y être.

À suivre Reinach, cette analyse de l'espace parcouru par le mouvement est généralisable à la durée occupée par le mouvement. Le problème ci-dessus réside dans le fait suivant : « $\mathrm{Au}$ premier moment du mouvement, le corps n'est pas au point de départ ; il n'y est pas encore » ( $W B$, p. 585). Ce qui, note-t-il, semble violer le principe de non-contradiction. Mais la contradiction est seulement apparente et tient à une « ambiguïté du concept d'être » : « Outre 1' "être" (= se trouver, demeurer) en un point, il y a encore d'autre manières possibles de se rapporter à un point : l'atteindre, le quitter, passer par lui. » (WB, p. 585.) En d'autres termes, le corps n'est pas au point $A$ ou à l'instant $t$ si « être » signifie « être en repos », mais il y est 
au sens où il y passe. Au sens large donc, au sens où « le devenir n'est pas un non-être » ( $W B$, p. 586), le corps est bien au point $A$ ou à l'instant $t$. Inversement, il est — au sens étroit — à l'instant $t$ ' où le mouvement s'arrête, mais $t$ ' ne peut plus être le dernier instant de son devenir. Le mouvement ne peut pas plus avoir de dernier moment que la distance parcourue ne peut avoir de point final :

Il y a un premier moment du mouvement : celui-ci est présent au moment où il commence (...). Par contre, il $n$ 'y a pas de dernier moment du mouvement. Il y a une fin du mouvement et un premier moment du repos. À chaque moment avant cela il y a du mouvement, à chaque moment après cela il y a du repos. (WB, p. 583.)

\section{L'analyse des apories de Zénon}

Examinons maintenant les apories de Zénon sur le mouvement. Reinach les ramène aux trois problèmes suivants (que je formule de façon légèrement différente) :

(1) Un corps en mouvement d'un point $A$ à un point $B$ devrait parcourir un nombre infini d'espaces — ce qui est impossible en un temps fini.

(2) Pour rattraper la tortue, Achille doit parcourir la distance qui l'en sépare. Mais pendant qu'il parcourt cette distance, la tortue doit avoir parcouru une distance supplémentaire. En conséquence, il est impossible à Achille de rattraper la tortue.

(3) À chaque instant de son parcours, la flèche en vol doit être en chaque point par où elle passe. Autrement dit, elle doit être au repos à chaque instant de son mouvement. Comme on ne peut pas être à la fois en repos et en mouvement, l'idée même de mouvement est contradictoire.

Reinach estime que les distinctions passées en revue dans la section précédente rendent possible une solution satisfaisante des trois apories. Comme le texte présente cependant moins une solution que des indications sur la méthode à suivre, il sera nécessaire d'extrapoler quelque peu ses conclusions.

Les apories (1) (2) — le corps parcourant un nombre infini d'espaces, Achille et la tortue posent des problèmes très semblables. D'un côté, il est impossible à un corps d'être en mouvement du fait qu'il devrait parcourir un nombre infini d'espaces. Par exemple, il devrait 
parcourir la moitié de l'espace entre deux points $A$ et $B$, puis la moitié de l'espace restant, c'est-à-dire le quart de la distance $A B$, puis à nouveau le huitième de la distance $A B$, et ainsi de suite à l'infini. De l'autre côté, Achille ne peut rattraper la tortue au point $A$ parce que la tortue a toujours parcouru une certaine distance pendant qu'il parcourait la distance le séparant de $A$. Achille franchit la distance $A C$ pendant que la tortue franchit la distance $C D$, puis la distance $C D$ pendant que la tortue franchit la distance $D E$, et ainsi de suite à l'infini.

Reinach relève trois points suscitant des « réserves ». D'abord, le mobile est censé passer par un nombre infini de points. Ensuite, il est censé parcourir un nombre infini de distances. Enfin, s'agissant d'Achille et de la tortue, « le fait de rattraper quelque chose serait spatialement inconcevable » $(W B$, p. 586).

Commençons par le premier point : le mobile doit passer par un nombre infini de points. Reinach ne conteste pas que le corps en mouvement de $A$ à $B$ passe par un nombre infini de points, tout comme il passe par un nombre infini de distances. Mais il ajoute que ce n'est pas vraiment un problème, ou plutôt que ce serait un problème seulement si le mobile devait être à chaque point plutôt que passer par chaque point. Car si le mobile est en un point déterminé $C$, alors il est forcément séparé de $A$ par une distance déterminée $A C$ - ce qui, comme on va le voir un peu plus loin, débouche nécessairement sur une impossibilité. Autrement dit, il est impossible qu'en allant de $A$ à $B$, le mobile s'arrête à chaque point de $A B$ de telle manière que chaque point soit le point final d'une distance partielle déterminée.

Cette idée est liée à celle, aristotélicienne, suivant laquelle un continuum n'est pas un complexe composé de parties ponctuelles (continuum non habet partes in actu, sed solum in potentia ${ }^{15}$. Brentano avait de même objecté à Renouvier et à Boltzmann, comme avant lui Aristote à Zénon, que l'existence d'un continuum n'implique pas celle d'une pluralité infinie de parties ${ }^{16}$. La possibilité de diviser ad libitum une ligne donnée en segments limités par des points n'implique pas qu'elle est composée d'une infinité de points (existant actuellement). Reinach reprend clairement à son compte cette conception dans le cours sur le mouvement. Il souligne que le continuum, à la différence des composés proprement dits, n'est pas fondé dans ses parties ponctuelles ${ }^{17}$. À l'inverse, les parties ponctuelles sont dépendantes du continuum. Elles ne sont pas des parties proprement dites, des « composantes » (Komponente, Bestandteile), mais plutôt des limites dégagées arbitrairement pour définir des continua partiels. C'est de cette manière, pour Reinach, qu'il convient de comprendre le fait que le continuum est divisible à l'infini.

Le deuxième point était que, pour aller de $A$ à $B$, le mobile doit franchir un nombre infini de distances. En vue de clarifier ce point, Reinach commence par envisager deux possibilités : d'une part la distance $A B$ est « couverte »; d'autre part elle est « parcourue ».

Supposons d'abord qu'elle est « couverte ». Cela signifie, on l'a vu, que le mouvement est achevé ou qu'il a atteint son but. La question sous-jacente aux deux premières apories est 
alors la suivante : est-il possible au corps en mouvement d'atteindre son but? Si le corps a atteint son but, c'est-à-dire s'il a « couvert » la distance $A B$, alors « il doit se trouver au point final d'une distance ». Reinach nous demande ensuite de considérer trois hypothèses, qui en excluent toute autre possible : premièrement le point final de la distance — appelons-le $F$ se situe avant $B$; deuxièmement il se situe après $B$; troisièmement il est identique à $B$.

Le raisonnement de Reinach est de montrer que les trois hypothèses conduisent à des impossibilités. La première, observe-t-il, est manifestement en contradiction avec l'hypothèse de départ : si le point final $F$ se situe avant $B$, alors le mouvement n'a pas atteint son but, puisque son but est $B$. Mais il en va de même de la deuxième hypothèse. Si le point final $F$ se situait après $B$, alors, observe Reinach, « il n'y aurait plus aucune distance partielle de $A B$ qui serait couverte en tant que dernière distance partielle » (WB, p. 587), ce qui est visiblement absurde. Le point final $F$ serait-il dès lors, comme le veut la troisième hypothèse, le point $B$ lui-même ? Ici encore, Reinach considère que l'hypothèse débouche sur une impossibilité. Car le point $B$ devrait être « le point final de toutes les distances ». La troisième hypothèse dit en effet que si le corps « couvre » la distance $A B$, alors il doit couvrir un nombre infini de distances partielles de telle manière que la dernière distance partielle ait $B$ pour point final. Mais il est tout simplement impossible que le corps « couvre » un nombre infini de distances partielles. Par conséquent, il est également impossible que le point $B$ soit le point final de la dernière distance partielle.

Pour comprendre l'argument de Reinach, il suffit de se rappeler nos analyses précédentes sur la structure du processus consistant à « couvrir » (zurücklegen) une distance. Si le corps couvre la première distance partielle $A C$, alors le mouvement doit s'interrompre au point $C$ en sorte que le point $C$ doit être le début d'un état de repos. Et il doit en être de même de la distance partielle suivante $C D$, puis de $D E$, et ainsi de suite à l'infini. Or, observe Reinach, tout point d'arrêt a une durée, si petite soit-elle : « Le repos comme le mouvement exigent une durée et ne sont pas pensables en un moment isolé. » ( $W B$, p. 586.) En d'autres termes :

\footnotetext{
L'interruption n'agrandit pas l'espace de mouvement, mais bien la durée de mouvement. Il doit y avoir un autre moment temporel où le mouvement cesse, différent du moment où il s'amorce à nouveau. Mais entre deux moments il y a toujours une durée, aussi petite soitelle. Ou, pour le dire autrement : dans la mesure où entre deux phases de mouvement s'intercale au point $C$ un état de repos, il faut ici une durée temporelle plus longue pour couvrir la distance $A B$. (WB, p. 559.)
}

Bref, si l'on considère que le mobile « couvre » chaque distance partielle, alors il faut une durée infinie pour couvrir $A B$, puisque $A B$ présenterait du même coup un nombre infini de distances partielles. Dans ce cas donc, il est impossible que $B$ soit le point final du mouvement de $A$ à $B$. 
Comme les trois hypothèses excluent toute autre hypothèse possible, la démonstration qu'elles conduisent à des impossibilités revient à réfuter l'hypothèse suivant laquelle la distance $A B$ est « couverte » par le corps en mouvement de $A$ à $B$. Mais si la distance $A B$ n'est pas « couverte », c'est donc qu'elle est « parcourue ». Pour Reinach, l'erreur de Bergson a été de comprendre les apories de Zénon au sens du « couvrir » et non du « parcourir ». Bergson a fait comme si le mobile devait s'arrêter à chaque point de $A B$, ou au point final de chaque distance partielle composant $A B$.

Qu'arrive-t-il maintenant si, comme le fait Reinach de manière conséquente, l'on décrète que la distance est « parcourue » et non « couverte » ? Alors, le mobile doit passer sans interruption par tous les points de $A$ à $B$, c'est-à-dire s'y trouver de telle manière qu'il n'est en aucun point de $A B$ et que la distance qui le sépare de $A$ n'est jamais une distance déterminée, mais un apeiron. On vient de voir que c'était impossible dans le cas où la distance $A B$ est « couverte ». Si elle est « couverte », alors il est impossible de passer par tous les points de $A B$ : en l'occurrence, le point final $B$ ne sera jamais atteint. Comme l'indique Reinach,

on peut passer par l'ensemble de tous les points d'une distance. On passe alors par plus de points encore (<qu'il n'y en a dans la distance couverte>, puisqu'il faut passer par le dernier point de la distance). Dans ce cas nous disons : la distance a été parcourue. (WB, p. $582^{18}$.)

Supposons donc que le mobile passe par tous les points de $A$ à $B$. Il passe donc par $B$ et en ce sens toute la distance $A B$ est parcourue : il est donc possible d'aller de $A$ à $B$. Mais on se heurte à une difficulté, car il semble alors que le point $B$ ne peut plus être le terme du mouvement. Le mobile ne peut plus s'arrêter au point $B$, puisqu'il doit au contraire passer par le point $B$, c'est-à-dire y parvenir et le laisser derrière lui. Sinon, la distance $A B$ est « couverte », et l'on se heurte aux mêmes impossibilités mentionnées plus haut.

Telle est la conclusion — paradoxale — à laquelle aboutit Reinach : il est certes possible de passer par tous les points de la distance $A B$, mais à la condition que le mouvement ne s'arrête pas au point $B$ et donc que $B$ ne soit pas le dernier point atteint par le mobile.

Au nombre infini de points par lesquels il faut passer, déclare-t-il, correspondent un nombre infini de moments dans la durée finie du processus de mouvement. Si l'on a préalablement en vue les points remarquables par lesquels il faut passer $(1 / 2,1 / 4,1 / 8,1 / 16$, etc.) et si l'on dit qu'aucun d'eux ne peut être le dernier, alors il faudra répliquer ceci : aucun dernier moment n'est présent. Et pourtant on passe par tous les points. La présupposition d'après laquelle il faudrait passer par un dernier point est fausse. ( $W B$, p. 586.)

Ou encore : 


\begin{abstract}
Bien entendu, si l'on saisit les choses de telle manière que seule la distance $A B$ doit être parcourue et rien de plus, alors ici aussi une solution est impossible : si le corps doit parcourir toutes les distances, il doit aussi passer par tous les points qui font partie de chacune des distances. Étant donné qu'il n'y a ni premier ni dernier point par où passer, $B$ ne peut pas davantage être le point final par où on passe. Comme pour tout point par où on passe, il y a également dans le cas de $B$ des points encore ultérieurs. Mais si on ne passait plus par $B$ lui-même, la distance totale ne serait donc pas non plus parcourue. Il faut donc qu'on passe par $B$ et, partout où l'on passe par un point, qu'on passe par un nombre infini de points ; ainsi le corps ne peut faire halte en $B$, mais il doit le dépasser. Partant, il ne lui est possible de parcourir la distance $A B$ que s'il lui est permis de parcourir plus encore. ( $W B$, p. 587.)
\end{abstract}

L'argumentation de Reinach peut ainsi être récapitulée de la manière suivante. D'abord, le mouvement ne peut s'arrêter à un nombre infini de points : « Le mouvement peut aussi s'arrêter en chemin, et cela à autant d'endroits qu'on veut. Mais ce serait un contresens de le faire s'arrêter à tous les points de la distance. » (WB, p. 582.) Toutefois, quoi qu'en pense Bergson, cette impossibilité n'est pas celle visée par Zénon. Ensuite, il reste possible d'aller de $A$ à $B$, mais à la condition que la distance $A B$ soit parcourue et non couverte, c'est-à-dire à la condition de passer par tous les points sans jamais s'y arrêter — de passer par tous les points $y$ compris $B$ - ce qui implique que $B$ ne peut être le point où s'achève le mouvement.

On peut facilement transposer ces résultats à l'aporie d'Achille et la tortue, quoique la situation soit un peu plus complexe. C'est ce que fait Reinach de façon conséquente dans les dernières lignes du texte sur le mouvement. Il commence par appeler « points équivalents » les points franchis par Achille et la tortue qui se trouvent à égale distance du point de départ $A$. Si la tortue a une avance sur Achille, alors on a, au début de mouvement, deux points non équivalents. Achille et le tortue ne passent par des points équivalents qu'au moment où Achille rattrape la tortue, puis à nouveau on a des points non équivalents dans la mesure où c'est désormais Achille qui a une avance. Mais une fois encore, la situation est incompréhensible si l'on comprend par là qu'Achille doit « couvrir » la distance qui le sépare de la tortue. Car alors il doit couvrir un nombre infini de distances déterminées dans une durée finie. Ce qui se produit plutôt, déclare Reinach, c'est que toutes les distances entre les points équivalents ou non équivalents sont « des apeira de grandeurs différentes » :

À toute distance temporelle limitée par deux moments correspondent des apeira de grandeurs différentes, si petite que soit la distance temporelle choisie. À aucun instant du mouvement l'avance n' $a$ une grandeur déterminée, et à tout instant le corps passe par une grandeur. ( $W B$, p. 588.) 
La même conclusion est formulée de façon un peu différente dans la brève remarque conclusive, issue de notes manuscrites, que Stein a insérée à la fin du cours sur le mouvement. Reinach introduit ici la notion de « pré-condition » (Vorbedingung). L'idée est d'abord qu'il existe une pré-condition nécessaire pour qu'Achille rattrape la tortue. Si au départ Achille se trouve au point $A$ et la tortue au point $B$, alors Achille doit parvenir au point $B$. Mais, observe Reinach, c'est là une pré-condition nécessaire et non suffisante, car la tortue a entre-temps atteint le point $C$. Dès lors apparaît une nouvelle pré-condition nécessaire qui est de parvenir au point $C$ et qui, à son tour, n'est pas suffisante parce que la tortue est parvenue entre-temps au point $D$, et ainsi de suite à l'infini. Le caractère aporétique de la situation décrite par Zénon vient du fait que de nouvelles pré-conditions à remplir s'ajoutent perpétuellement aux préconditions remplies. Chaque pré-condition impose nécessairement, une fois remplie, une nouvelle pré-condition :

Le caractère insoluble du problème de Zénon repose sur le fait qu'il est dans l'essence de la pré-condition remplie de poser une nouvelle pré-condition, et qu'aucune pré-condition ne peut donc être la dernière. ( $W B$, p. 588.)

De toute évidence, c'est là une manière différente de dire la même chose. Ce qui est aporétique dans le cas des distances parcourues, c'est qu'il faut apparemment passer par le dernier point, mais que, pour passer par le dernier point, il est nécessaire de parvenir au point suivant, et que le dernier point ne peut donc pas être, à proprement parler, le dernier point. En ce sens, le parcours spatial ajoute toujours une nouvelle pré-condition qui est de parvenir à un point encore ultérieur.

\section{Remarques conclusives : Reinach et Brentano}

Le point de vue de Reinach peut assurément sembler outrancièrement réaliste, le conduisant à défendre, par exemple, l'idée d'un mouvement absolu contre la théorie de la relativité ${ }^{19}$. Il apparaît pourtant moins sujet à caution si l'on perçoit correctement l'ambition de Reinach, qui est fondamentalement, comme il l'indique aux premières lignes du $\S 3$, de « se porter vers l'essence pure des choses mêmes » tandis que « d'autres sciences saisissent les choses sous des points de vue déterminés et en opérant une sélection en fonction des buts visés » ( $W B$, p. 561). Au-delà d'une impression de platonisme indigeste, l'essentiel de l'entreprise de Reinach serait peut-être préservé et rendu moins déroutant si, au lieu de clarification des essences, on parlait d'analyse grammaticale ou conceptuelle. Par exemple, la question n'est 
pas de savoir s'il existe ou non des mouvements absolus ou relatifs, mais de savoir s'il y a un sens à parler de mouvement absolu ou relatif et, plus largement, ce qui est impliqué conceptuellement dès lors qu'on parle de «mouvement ». Ainsi compris, le projet de Reinach serait peut-être moins éloigné de celui de Brentano qu'il ne peut sembler à première vue ${ }^{20}$.

Le texte de Reinach de 1913-1914 - comme celui de Koyré de 1922 — ne contient aucune référence explicite à Brentano. À supposer que Reinach ait eu accès à la théorie brentanienne du continu, il serait difficile de déterminer dans quelle mesure et par quels canaux. Mis à part quelques indications éparses, les publications de Brentano sur le continu ne remontent pas audelà de $1924^{21}$. Les deux conceptions présentent néanmoins des similitudes significatives. Très généralement, Reinach comme Brentano font jouer à la temporalité un rôle central et rejettent la conception mathématique du continu de Cantor et Dedekind. D'autres convergences sont seulement partielles. Brentano, on l'a vu, définissait le continu d'une part par l'impossibilité que des points, instants, etc., soient contigus (la ligne n'est pas composée de points comme elle est composée de lignes plus petites), d'autre part par la possibilité de la coïncidence (deux lignes contiguës ont une limite commune). Reinach, dans sa théorie du changement continu, s'accorde avec Brentano sur le premier point : " Il n’y a pas de points contigus, puisque deux endroits (Stellen) d'un continuum limitent une partie, si petite fûtelle. » $(W B$, p. 582, n.) En revanche, il rejette l'idée d'une coïncidence entre la fin de l'état de repos et le début de l'état de mouvement, ou entre la fin de l'état de mouvement et le début de l'état de repos : un corps ne peut pas à la fois passer par un point et y être. D'où il suit que ni le mouvement ni le repos n'ont de dernier moment. À plus forte raison, ils n'auront pas non plus d'avant-dernier moment, c'est-à-dire de moment contigu au dernier moment (cf. $W B$, p. 582, n.).

Il est important de garder à l'esprit, cependant, que les deux théories n'ont pas le même degré de généralité. Ainsi les analyses reinachiennes de l'espace parcouru ne sont pas généralisables sans plus au continuum temporel. Lorsqu'un corps interrompt son mouvement en $B$, dit Reinach, il est au point $B$ plutôt qu'il ne passe par lui. Mais le corps n'en passe pas moins par l'instant correspondant. Le temps semble un continuum qui n'admet pas d'interruption. Reinach parle en ce sens de « continuum primaire », par opposition aux « continua secondaires » comme l'espace parcouru ${ }^{22}$. Cette distinction en implique une autre entre le temps en général et la durée occupée par un processus ${ }^{23}$. Quand le mouvement s'interrompt, sa durée s'interrompt également. Par exemple d'un individu qui marche deux heures, fait une pause de deux heures, puis marche à nouveau deux heures, on dira qu'il a marché quatre heures. Mais le temps aura poursuivi son cours sans interruption, et l'on dira avec raison qu'il y a du temps entre les deux marches de deux heures. Une constatation analogue, note Reinach, peut être faite au sujet de l'espace en général par opposition à la trajectoire parcourue, et des continua intensifs (par exemple celui des intensités sonores) par opposition aux gradations intensives (tel ou tel accroissement sonore). Or, l'essentiel des analyses de 
Reinach dans le cours de 1913-1914 porte sur des continua secondaires, ce qui n'est pas le cas de la théorie brentanienne. Ce qui laisse penser qu'une part non négligeable de celle-ci - y compris, comme je l'ai suggéré dans la section précédente, la définition du continu en termes de limites - est peut-être intégrable dans un cadre reinachien.

Plus généralement, il est clair que les deux théories trouvent leur origine sinon dans une réaction contre une certaine conception mathématique du continu, du moins dans la volonté de la doter de fondations intuitives. Chez Brentano, l'opposition à Cantor et Dedekind s'explique avant tout par son approche intuitionniste ou phénoménologique du continu ${ }^{24}$. Au lieu de voir dans le continu une construction abstraite, l'enjeu est de rendre compte de sa phénoménalité. Ce n'est pas seulement qu'il est possible d'avoir l'expérience du continu. Plus encore, observe Brentano, le continu caractérise essentiellement tout ce qui nous est donné dans l'expérience : « Toutes nos intuitions, aussi bien celles de la perception externe que celles de la perception interne qui les accompagnent et par suite aussi celles du souvenir, font apparaître du continu ${ }^{25}$. » Partant, un objectif majeur de la théorie brentanienne du continu est d'établir que le continu est un concept « tiré immédiatement d'une intuition » plutôt qu'une construction résultant d'une combinaison de caractères empiriques ${ }^{26}$. Or, l'idée d'une expérience du continuum est absurde si le continuum se définit comme étant composé d'une infinité de parties ponctuelles.

Bien que très différente, l'approche de Reinach dans le cours sur le mouvement obéit à un principe analogue ${ }^{27}$. Son point de départ est la réponse de Diogène le Cynique à Zénon : Diogène se lève sans mot dire et fait quelques pas devant Zénon (Diogène Laërce, VI, 39). Le contre-argument ne prouve pas l'existence du mouvement, car l'expérience peut toujours être trompeuse. Mais elle démontre néanmoins, poursuit Reinach en songeant plausiblement à la notion de « contresens » de la $\mathrm{IV}^{\mathrm{e}}$ Recherche logique de Husserl ${ }^{28}$, la possibilité a priori du mouvement. Si tout ce qui est intuitionnable est possible a priori, alors l'intuition du mouvement suffit à invalider la démonstration zénonienne de l'impossibilité a priori — du caractère contradictoire - du mouvement. Reinach poursuit cependant ses réflexions dans une voie nouvelle, qu'il qualifie d' « ontique » (WB, p. 554). Toute impossibilité a priori, clame-t-il, trahit une relation d'incompatibilité entre des essences, entre des « quiddités » (Washeiten), qui sont accessibles à l'intuition eidétique. Pour autant qu'elles ont pour enjeu la possibilité idéale du changement continu, les apories de Zénon appellent un traitement philosophique au moyen de l'intuition des essences :

L'essentiel doit être de se transporter dans l'essence des éléments en question que sont le mouvement et le changement, de se libérer de tous les concepts et définitions qui nous empêchent de voir ce qui leur est propre, et d'établir, par une intuition juste, inaltérée du mouvement et du changement eux-mêmes, ce qui se fonde en eux d'après des lois d'essence. (WB, p. 556-557.) 


\section{Notes}

${ }^{1}$ A. Reinach, «Über das Wesen der Bewegung », Sämtliche Werke : Textkritische Ausgabe, éd. K. Schuhmann \& B. Smith, München, Philosophia Verlag, 1989, vol. 1, p. $551-588$ (désormais WB). Trad. fr. D. Seron dans Id., Phénoménologie réaliste, Paris, Vrin, 2012, p. 281-325.

${ }^{2}$ Alexandre Koyré partait d'une constatation analogue dans son étude de 1922 sur les paradoxes de Zénon (voir infra). Il ajoutait cependant que le texte de Reinach — qu'il connaissait par les Gesammelte Schriften, parues l'année précédente - a trait principalement au mouvement et, curieusement, qu'il « ne traite qu'en passant des apories de Zénon ». Voir A. Koyré, « Bemerkungen zu den Zenonischen Paradoxien », Jahrbuch für Philosophie und phänomenologische Forschung, 5, 1922, p. 601 et 628.

${ }^{3}$ Pour la suite, cf. la notice critique de Schuhmann et Smith dans Sämtliche Werke, vol. 2, p. 775-778.

${ }^{4}$ H. Weyl, Das Kontinuum. Kritische Untersuchungen über die Grundlagen der Analysis, Leipzig, Veit \& Comp., 1918, p. 2.

${ }^{5}$ H. Lipps, « Die Paradoxien der Mengenlehre », Jahrbuch für Philosophie und phänomenologische Forschung, 6 (1923), partant des antinomies du Menteur, de Russell et de Nelson et Grelling. A. Koyré, «Bemerkungen zu den Zenonischen Paradoxien », art. cit. Cf. P. Zambelli, «Alexandre Koyré im "Mekka der Mathematik" », NTM Zeitschrift für Geschichte der Wissenschaften, Technik und Medizin, 7/1 (1999), p. 208-230. Sur H. Lipps, ibid., p. 212. Husserl a consacré plus tard quelques notes aux antinomies logiques (manuscrit A I 35, daté de 19181927, dont un fragment consacré à un autre sujet a été publié dans Natur und Geist. Vorlesungen Sommersemester 1927, éd. M. Weiler, Hua 32, p. 210-216).

${ }^{6}$ De même Koyré, art. cit., p. 626, qui nuance cependant cette lecture de Bergson, ibid., p. 611-612.

${ }^{7}$ G. Berkeley, A Treatise concerning the Principles of Human Knowledge, § 112, cité dans WB, p. 563.

${ }^{8}$ La conception incriminée ne sera publiée que deux ans plus tard, P.F. Linke, «Phänomenologie und Experiment in der Frage der Bewegungsauffassung », Jahrbuch für Philosophie und phänomenologische Forschung, vol. 2 (1916). Il est très vraisemblable que Reinach en ait pris connaissance à une date antérieure en qualité de co-éditeur de la revue. Cf. A. Reinach, Sämtliche Werke, vol. 2, p. 777.

${ }^{9}$ A. Reinach, «Zur Theorie des negativen Urteils », Sämtliche Werke, vol. 1, p. 95 suiv.

${ }^{10}$ R. Ingarden, Der Streit um die Existenz der Welt, Tübingen, Niemeyer, 1964, vol. 1, p. 191 suiv. R. Chisholm, «Events without times : An essay on ontology », Noûs, 24/3 (1990). Id., A Realistic Theory of Categories : An Essay in Ontology, Cambridge University Press, 1996, p. 73 suiv. K. Mulligan et B. Smith, «A relational theory of the act », Topoi, 5/2 (1986), ont développé une variante de cette distinction en termes de limites de continua, inspirée de la théorie brentanienne du continu.

${ }^{11}$ Comme le fait pourtant très naturellement Stein dans sa note, $W B$, p. 583. 
${ }^{12}$ F. Brentano, Philosophische Untersuchungen zu Raum, Zeit und Kontinuum, éd. par St. Körner \& R. Chisholm, Hamburg, Meiner, 1976, p. 40-41, 217-218. Cf. R. Chisholm, « Beginnings and endings », dans Id., Brentano and Meinong Studies, Amsterdam, Rodopi, 1982, et E. Fugali, Die Zeit des Selbst und die Zeit des Seienden : Bewusstsein und innerer Sinn bei Franz Brentano, Würzburg, Königshausen \& Neumann, 2004, chap. 4, qui l'un et l'autre exploitent les inédits.

${ }^{13}$ R. Chisholm, « Beginnings and endings », art. cit., p. 117.

${ }^{14}$ A. Varzi, Ontologia, Roma-Bari, Laterza, 2005, p. 129.

${ }^{15}$ Thomas d'Aquin, In De caelo, lib. 1, lect. 3. Pour la suite, voir WB, p. 574 suiv.

${ }^{16}$ F. Brentano, Psychologie vom empirischen Standpunkt, vol. 2 : Von der Klassifikation der psychischen Phänomene, Leipzig, Meiner, 1925, p. 255-256.

${ }^{17} \mathrm{Cf}$. la distinction de Husserl entre synthèses continues et articulées (ou discrètes), dans Logische Untersuchungen, 6, A570 et A619 suiv., Hua 19/2, p. 628 et 676 suiv., ainsi que Ideen I, Hua 3, § 118.

${ }^{18}$ Je complète l'adjonction de Stein, manifestement lacunaire.

${ }^{19}$ Brentano s'oppose à la théorie de la relativité pour d'autres motifs, qui tiennent à sa distinction entre continua primaires et secondaires. Cf. F. Brentano, Philosophische Untersuchungen zu Raum, Zeit und Kontinuum, op. cit., p. 29-30, et K. Hedwig, « Brentano und Kopernikus », dans M. David \& L. Stubenberg (éds.), Philosophische Aufsätze zu Ehren von Roderick M. Chisholm, Amsterdam, Rodopi, 1986, p. 253-274.

${ }^{20}$ Pour une lecture de Brentano en ce sens (par opposition à Meinong), voir F. Boccaccini, « The bounds of object : The Brentano-Meinong dispute, a priori knowledge, and the power of perception », à paraître.

${ }^{21}$ F. Brentano, Aristoteles und seine Weltanschauung (1911), Hamburg, Meiner, 1977, p. 36-37, sur le continu et les apories de Zénon. Les premières réflexions sur le continu semblent remonter à la fin des années 1870 (voir E. Fugali, Die Zeit des Selbst und die Zeit des Seienden, op. cit., p. 168). Achevant à cette époque son doctorat à Munich, Reinach ne semble pas avoir assisté aux leçons de Husserl sur le temps de 1904-1905. Voir K.

Schuhmann \& B. Smith, « Adolf Reinach : An intellectual biography », dans K. Mulligan (éd.), Speech Act and Sachverhalt : Reinach and the Foundations of Realist Phenomenology, Dordrecht, Nijhoff, 1987, p. 7-8.

${ }^{22}$ WB, p. 577 : « Nous qualifierons de primaires les continua qui — comme l'espace et le temps — ne permettent pas d'interruption, et de secondaires les autres. » La distinction n'est pas très éloignée de celle de Brentano, y compris par l'idée que « les continua secondaires sont fondés dans les continua primaires » (ibid.). Cf. F. Brentano, Philosophische Untersuchungen zu Raum, Zeit und Kontinuum, op. cit., p. 28 et 219 ; Id., Untersuchungen zur Sinnespsychologie, éd. R. Chisholm \& R. Fabian, Hamburg, Meiner, 1979, p. 199 suiv.

${ }^{23}$ Cf. E. Fugali, Die Zeit des Selbst und die Zeit des Seienden, op. cit., p. 192 suiv.

${ }^{24}$ Cf. S. Körner \& R. Chisholm, « Einleitung », dans F. Brentano, Philosophische Untersuchungen zu Raum, Zeit und Kontinuum, op. cit., p. xii-xv, et Id., « Editors' Introduction to English Edition », dans F. Brentano, Philosophical Investigations on Space, Time and the Continuum, trad. angl. B. Smith, Croom Helm, 1988, p. ixxi. De là une proximité intéressante avec des mathématiciens comme H. Weyl, Das Kontinuum, op. cit., lequel, sous l'influence de Husserl, oppose à Cantor et Dedekind l'idée d'une donation intuitive du continuum arithmétique. Cf. l'analogie avec le « temps phénoménal », ibid., p. 67 suiv.

${ }^{25}$ F. Brentano, Philosophische Untersuchungen zu Raum, Zeit und Kontinuum, op. cit., p. 9.

${ }^{26}$ Ibid., p. 3. 
${ }^{27}$ En dépit de son style bergsonien (par la distinction entre continuum et grandeur continue), l'argumentation de Koyré contre Bolzano et Cantor dans ses « Bemerkungen zu den Zenonischen Paradoxien », art. cit., élude en revanche totalement la question de la phénoménalisation du continu.

${ }^{28}$ E. Husserl, Logische Untersuchungen, 4, § 12, A312-313, Hua 19/1, p. 334-336. 